European Journal of Social Psychology

Eur. J. Soc. Psychol. 29, 1011-1020 (1999)

\title{
Telling what they want to know: participants tailor causal attributions to researchers' interests
}

\author{
ARA NORENZAYAN* and \\ NORBERT SCHWARZ \\ University of Michigan, USA
}

\section{Abstract}

Based on a conversational analysis of experimental procedures and consistent with the principle of relevance, we predicted that participants' verbal responses will be influenced by their tacit inferences about the researcher's epistemic goals, derived from their knowledge of the researcher's academic affiliation. We tested this prediction in a core area of social-personality and cultural psychology, causal attribution. University students provided causal attributions about mass murder cases, while the questionnaire identified the researcher either as a social scientist or a personality psychologist. The results indicated that attributions were overall more situational than dispositional, and as predicted, this main effect was qualified by an interaction between conversational cue and type of attribution. Thus, participants gave relatively more situational explanations when the letterhead of the questionnaire identified the researcher as a social scientist compared to when the researcher was identified as a personality psychologist. The reverse pattern emerged for dispositional attributions. Methodological and conceptual implications are discussed. Copyright (C) 1999 John Wiley \& Sons, Ltd.

A common research strategy in psychology is to ask participants to generate verbal answers in response to questions asked by the researcher. For example, participants may be asked to explain the behavior of a target person in a causal attribution task (e.g. Miller, 1984; Morris \& Peng, 1994) or to describe themselves (e.g. Cousins, 1989; McGuire \& McGuire, 1988). These responses are then content-analyzed based on some well-established categories, such as situational versus dispositional attributions or social versus psychological self-descriptions. Psychologists assume that this methodology allows them to probe into salient aspects of participants' attributional style or self-concepts. The present research demonstrates that participants' verbal

*Correspondence to: Ara Norenzayan, Department of Psychology, University of Michigan, 525E. University Avenue, Ann Arbor, MI 48109, USA. e-mail: anorenz@umich.edu

Contract grant sponsor: US National Science Foundation.

CCC 0046-2772/99/081011-10\$17.50

Copyright (C) 1999 John Wiley \& Sons, Ltd. Accepted 23 September 1998 
responses to such tasks are partially a function of their tacit inferences about the researcher's epistemic goals, which can be derived from knowledge of the researcher's academic affiliation.

A central tenet governing everyday conversation is the principle of relevance (Grice, 1975; Sperber \& Wilson, 1995). According to the relevance principle, 'the speaker tries to make his utterance as relevant as possible to the hearer. The hearer has a systematic expectation of relevance' (Sperber \& Wilson, 1981, p. 284). Specifically, speakers are supposed to provide information that is informative in the particular context of the ongoing conversation, requiring complex inferences about the listeners' respective interests and the intended meaning of their questions. In everyday conversations, these inferences are typically based on previous utterances and assumptions about shared background knowledge. In research situations, participants may draw on features of the research instrument - such as the content of preceding questions, or the nature of the response alternatives - to infer what the researcher means and is interested in (see Schwarz, 1994, 1996, for reviews).

A related line of research examining the communicative context of cognitive processes has focused on audience characteristics. Social psychologists have long been aware that people tailor their message to the characteristics of the hearers (see McCann \& Higgins, 1992, for a review). These audience effects occur routinely in everyday conversations, where speakers may infer what listeners want to know from some aspect of their background. For example, in response to the question from a friend 'What's in the news lately?' the listener, knowing that the friend is an avid follower of the sports, might tell the friend about the latest college football match rather than recent developments in the Middle East peace process. Curiously, however, the implications of conversational inferences and response tailoring for the methodology of psychological research are often overlooked.

In this paper, we propose that participants can learn about the researcher's likely interests by exploiting a formal aspect of the research situation: the researcher's academic background. This may systematically bias the kinds of verbal responses participants provide, depending on who they believe the researcher is. We tested this hypothesis in a research domain of central concern to social-personality, as well as cultural psychology: causal attribution. Specifically, we hypothesized that participants implicitly assume that social scientists are interested in social contextual variables, whereas personality psychologists are interested in personality dispositions. To the extent that participants try to render their contributions relevant to the researcher's assumed epistemic goals, their attributions may focus on dispositional characteristics when the researcher is a psychologist, but on situational factors when the researcher is a social scientist.

\section{CAUSAL ATTRIBUTIONS AS A FUNCTION OF THE RESEARCHER'S ACADEMIC AFFILIATION}

Participants read about a mass murder case and were asked to explain why the murder occurred. Depending on conditions, the letterhead of the questionnaire informed participants that the researcher was affiliated with the 'Institute of Personality Research' or with the 'Institute of Social Research'. We predicted that 
participants would infer the researcher's likely epistemic interests from the letterhead and would tailor their attributional reports to be relevant to these interests. Hence, they would provide more situational and fewer dispositional attributions when the researcher is a social scientist rather than a personality psychologist.

Although we derived this prediction from a conversational relevance analysis, it is conceivable that the letterhead may prime different knowledge structures (see Higgins, 1996, for a review), an effect that may itself be sufficient to produce the expected results. Importantly, previous research has shown that priming effects occur at the encoding stage, rather than at the judgment or reporting stage (see Wyer \& Srull, 1989, for a review). In one study, for example, Srull and Wyer (1980) demonstrated that priming a trait concept before information about a target person was presented affected judgments of the target, but priming the same trait concept after the information was presented, had no effect on the subsequent judgments (see also Massad, Hubbard, \& Newston, 1979; Wyer, Srull, Gordon, \& Hartwick, 1982). Similarly, Bower, Gilligan, and Monteiro (1981) found that mood effects on recall of a passage occurred when the mood manipulation was introduced before presentation of the passage, but not when the manipulation came after the passage was already encoded by participants. Summarizing the literature on priming effects at the encoding versus reporting stages of social judgment, and consistent with their 'storage bin' model of social cognition, Wyer and Srull (1989, p. 145) concluded that 'postinformation processing objectives, unlike objectives that exist at the time information is first acquired, have very little influence on the interpretation and selective recall of this information once the information has been encoded and stored in memory'.

Accordingly, we varied the time at which participants learned about the researcher's affiliation. Some participants saw the institute letterhead before reading the story (Cue Before Story Condition); others first read the story, and then saw the institute letterhead prior to generating attributions (Cue After Story Condition). If the predicted effects are solely due to cognitive priming as construed by Wyer and Srull's (1989) model rather than to conversational inferences, they should only be obtained in the Cue Before Story Condition, but not in the Cue After Story Condition. If the predicted effects reflect response tailoring, however, they should be obtained under both conditions and may be more pronounced when the researcher's epistemic interests are rendered salient just prior to the response formulation.

\section{METHOD}

\section{Participants}

Participants were 60 students, 29 male and 31 female, (mean age 20.68 years), recruited at various gathering places on the University of Michigan campus.

\section{Materials}

Two real mass murder stories were used since highly negative behaviors tend to generate spontaneous attributional activity (Morris \& Peng, 1994). Half of the participants read a newspaper story about Thomas McIlvane, a disgruntled postal 
worker who went on a mass murder spree in a Royal Oak, Michigan, post office in 1991. The other half read about Timothy McVeigh, the man accused and later convicted of murder in the bombing of the Oklahoma Federal Building, which occurred in 1995. Both stories were taken from articles from the New York Times, and had about equal length.

\section{Design and Procedure}

The study followed a 2 (conversational cue: personality versus social) by 2 (order: cue before story versus cue after story) by 2 (story: post-office shooting versus Oklahoma bombing) factorial between-subjects design. Responses to either of the two stories served as the dependent variable.

The conversational cue was provided by printing the questionnaire on the letterhead of an alleged 'Institute of Personality Research' or an 'Institute of Social Research'. In the Cue Before Story condition, this letterhead was presented on the first page, preceding the mass murder story. In the Cue After Story condition, the letterhead was presented on the sheet that assessed the dependent variables. After participants had read one of the above murder stories, they were asked to list five reasons which they believed explained why the person in the story committed the crime.

\section{Coding of Responses}

Three coders, who were blind to the experimental conditions, rated the causal explanations, following a coding scheme similar to those of Miller (1984) and Morris and Peng (1994). Dispositional attributions are explanations that refer to factors stable and internal to the actor in the story: enduring psychological properties that the actor is thought to carry across time, place, and social context. These include personality traits, chronic pathology, stable temperament, long-standing and stable goals, values or attitudes, and character flaws. Situational attributions are explanations pointing to factors external to the actor in the story: properties that are tied to a particular time, place, or situation. These include the immediate, specific situation (e.g. stress in the workplace, a relationship, social role), or the larger social context (e.g. violence in society at large, group norms, the media, cultural practices). Explanations, which could not be readily coded as either situational or dispositional, were assigned to an Other category. The reliability across the three coders and two stories was high, Kendall's coefficient of concordance, $W=0.88, p<0.001$, for dispositional attributions, and $W=0.93, p<0.001$, for situational attributions.

\section{Dependent Measures}

Two dependent variables were created. First, based on the total number of reasons provided by a given participant, we calculated each participant's percentage of dispositional and situational attributions. This measure reflects the relative amount of situational and dispositional attributions (percentage measure). Second, we created two weighted attribution measures that reflect the relative amount of dispositional 
and situational attributions, as well as the order in which the respective attributions were listed (weighted measure). Specifically, we assigned the first explanation a score of 5 and the fifth explanation a score of 1 . Next we added the scores of all dispositional and all situational attributions to arrive at two separate weighted measures. A value of 0 indicates that no dispositional (or situational, respectively) attribution was provided, whereas a score of 15 indicates that only dispositional (or situational, respectively) attributions were listed. These weighted measures are somewhat more sensitive than the percentage measure because they capture the priority given to dispositional versus situational attributions, as reflected in the temporal order in which participants provided their attributions.

\section{RESULTS}

Table 1 shows the results in terms of the weighted measures, and Table 2 represents the mean percentage results. The top panels of both tables show the respective means collapsed over both cue order (i.e. letterhead before versus after the story) conditions. Our analyses treat participants' dispositional and situational attributions as two levels of a within-subjects factor (ignoring the 'Other' category). The statistical tests shown below were conducted on the weighted measures. However, as the means in Tables 1 and 2 indicate, the pattern of results for the percentage measure was very similar to that of the weighted measure.

Overall, participants provided more situational than dispositional attributions, and listed them earlier, $F(1,52)=14.84, p<0.001$, for the main effect of attributional category. More importantly, participants provided more situational attributions, and listed them earlier, when the letterhead identified the researcher as a social scientist $(M=8.7)$ rather than a personality psychologist $(M=5.8)$, as reflected in a significant simple effect of the researcher's affiliation, $F(1,52)=7.58, p<0.01$. Conversely, participants gave more dispositional attributions, and listed them earlier,

Table 1. Weighted measure: causal attributions as a function of conversational cue and cue order

\begin{tabular}{lccc}
\hline & & Attributions & \\
& Dispositional & Situational & Other \\
\hline $\begin{array}{l}\text { Conversational Cue } \\
\text { Overall }\end{array}$ & & & \\
$\quad$ Personality $(n=30)$ & 4.4 & 5.8 & 4.1 \\
$\quad$ Social $(n=30)$ & 2.6 & 8.7 & 3.1 \\
Cue After Story & & & \\
$\quad$ Personality $(n=15)$ & 4.5 & 5.8 & 2.1 \\
$\quad$ Social $(n=15)$ & 2.6 & 9.4 & \\
Cue Before Story & & & 4.2 \\
$\quad$ Personality $(n=15)$ & 4.4 & 5.8 & 3.8 \\
$\quad$ Social $(n=15)$ & 2.6 & 8.0 & \\
\hline
\end{tabular}

Notes: Personality = 'Institute of Personality Research'; Social = 'Institute of Social Research'. Weighted measure $=$ mean responses after being weighted according to retrieval order. 
Table 2. Percent measure: causal attributions as a function of conversational cue and cue order

\begin{tabular}{lccc}
\hline & & Attributions & \\
& Dispositional & Situational & Other \\
\hline $\begin{array}{l}\text { Conversational Cue } \\
\text { Overall }\end{array}$ & & & \\
$\quad$ Personality $(n=30)$ & 33.9 & 38.8 & 27.3 \\
$\quad$ Social $(n=30)$ & 19.7 & 57.7 & 24.6 \\
Cue After Story & & & \\
$\quad$ Personality $(n=15)$ & 32.5 & 40.2 & 18.1 \\
$\quad$ Social $(n=15)$ & 20.9 & 61.0 & \\
Cue Before Story & & & 27.4 \\
$\quad$ Personality $(n=15)$ & 35.2 & 37.4 & 31.1 \\
$\quad$ Social $(n=15)$ & 18.5 & 50.4 & \\
\hline
\end{tabular}

Notes: Personality = 'Institute of Personality Research'; Social = 'Institute of Social Research'. Percent measure $=$ mean percentage of responses.

when the letterhead identified the researcher as a personality psychologist $(M=4.4)$ rather than a social scientist $(M=2.6)$, again reflected in a significant simple effect of the researcher's affiliation, $F(1,52)=5.80, p<0.05$. Overall, this pattern resulted in the predicted significant interaction of conversational cue and type of attribution, $F(1,52)=8.08, p<0.01$.

Next, we address whether these overall effects depend on the time at which participants became aware of the researcher's affiliation. The second panels of Tables 1 and 2 show participants' attributions under conditions where the letterhead cue was provided after the story, immediately preceding the dependent variables. As expected, participants provided fewer dispositional and considerably more situational explanations when the letterhead identified the researcher as a social scientist rather than as a personality psychologist, $F(1,52)=4.95, p<0.05$, for the simple interaction. When the researcher's affiliation was provided prior to the story, a similar pattern was obtained, as shown in the bottom panels of Tables 1 and $2, F(1$, $52)=3.08, p=0.08$, for the simple interaction. Because of the similarity of these patterns, no triple interaction emerged, $F<1$. Finally, there was no effect of story version, nor any interactions with story version in these data.

\section{DISCUSSION}

In summary, participants overall gave more situational then dispositional explanations for the mass murder cases. This main effect, however, was qualified by the predicted interaction, such that participants gave relatively more situational attributions, and listed them earlier, when the letterhead of the questionnaire identified the researcher as a social scientist rather than as a personality psychologist. The reverse pattern emerged for dispositional attributions, such that participants gave relatively more dispositional explanations, and listed them earlier, when the letterhead identified the researcher as a personality psychologist rather than as a social scientist. 
This overall pattern was the same regardless of whether or not participants learned about the researchers' background before or after reading the story.

We propose that this 'letterhead effect' reflects the operation of the principle of relevance, leading participants to make their answers relevant to the likely epistemic goals of the researcher. People believe social scientists are interested in social and contextual explanations of human behavior, whereas personality psychologists are interested in chronic psychological characteristics as likely determinants of behavior. Thus, in an attempt to be cooperative communicators, participants try to provide information which they believe is relevant to the interests of the researchers: situational causes of behavior are more relevant to the interests of social scientists, whereas dispositional causes are more relevant to the interests of personality researchers.

Although our findings are consistent with the relevance principle of conversational conduct, it is worth noting that other conversational principles may affect the attributions that participants report in different ways. One such principle is the maxim of quantity (Grice, 1975), which invites speakers to avoid redundancy. This maxim encourages the provision of information that is new to the recipient and discourages the reiteration of information that the recipient already has. Consistent with the maxim of quantity, Slugoski, Lalljee, Lamb, and Ginsburg (1993) found that participants who were led to believe that their conversational partner shared only dispositional information about a target provided more situational explanations for the target's behavior. In contrast, those who believed that their partner shared only information about the situational background of the target gave somewhat more dispositional explanations. Thus, under conditions that resembled ordinary conversations, participants emphasized information that was likely to be new to the recipient and avoided reiterating information that was already part of the common ground. At first glance, one may therefore wonder why participants in the present study did not focus on explanations that the researcher may otherwise miss, that is, dispositional explanations when the researcher was allegedly a social scientist and situational explanations when the researcher was allegedly a personality psychologist.

We propose that two factors contribute to this apparent discrepancy between Slugoski et al.'s (1993) findings and the present research. First, the letterhead manipulation used in the present study did not convey information about differences in the common ground. Although the researcher may know more about the murder case than is conveyed in the newspaper story, the exact nature of any knowledge differences remains elusive, in contrast to the Slugoski et al. (1993) study, where the recipient was known to share either situational or dispositional information. To render redundancy avoidance a salient concern under the present conditions, one should lead participants to reason, 'as a personality psychologist, she certainly can think of personality explanations herself — so let me tell her about situational ones'. But no such concern was made salient in our study. Second, were participants to proceed along this line, they would have arrived at contributions that are likely to have been irrelevant to the epistemic agenda of the researcher, quite in contrast to Slugoski et al.'s (1993) experiment, where the epistemic agenda of the recipient remained unspecified. This is crucial, since research participants probably expect researchers to hold a certain degree of epistemic commitment corresponding to their fields' research agenda. Thus, people are likely to assume that someone becomes a sociologist because she is committed to learn about social explanations of behavior, 
and personality researchers are believed to be in the business of studying dispositional explanations of behavior.

Finally, two non-conversational accounts of the present findings deserve attention. One is a cognitive priming account (e.g. Wyer \& Srull, 1989). From this perspective, different letterhead cues may activate different knowledge structures, which may subsequently affect the encoding of the story, resulting in different prime-consistent explanations. Although knowledge-activation may have contributed to the overall effect, we believe that a mere priming interpretation cannot account for the entirety of the results. As several studies have demonstrated (see Wyer \& Srull, 1989, for a review), priming effects are obtained when the prime precedes the to-be-encoded information, but not when the prime follows this information. Hence, the present findings should only have been obtained when the letterhead cue preceded the story. Contrary to this prediction, the impact of the researcher's affiliation on participants' situational and dispositional attributions was unchanged (and if anything, became stronger) when the affiliation information was introduced after participants had read the story. Hence, participants were equally likely to tailor their responses to the researcher's epistemic interests when these interests were rendered salient after message encoding, and just before they formulated their answers, as would be expected from a conversational perspective.

Another account draws on Orne's (1962) concept of demand characteristics and assumes that research participants try to confirm the researcher's hypotheses. This account typically requires conversational inference processes to explain how participants infer the researcher's hypotheses in the first place (see Kihlstrom, 1995; Schwarz, 1996, for more detailed discussions). It differs from a conversational account, however, by postulating motivations that are specific to the role of research participants, and are driven by a desire to substantiate the researchers' expectations. In contrast, conversational analyses based on Grice's (1975) logic of conversation maintain that people strive to be cooperative communicators, emphasizing that cooperative conduct is a natural part of any conversation and not a peculiar aspect of experimental settings. As Kihlstrom (1995, p.11) noted, 'Subjects aren't just motivated to guess and confirm the experimenter's hypothesis. As listeners, that is, as people, they are primarily motivated to make sense of any communicative situation in which they find themselves.' From this perspective, research participants are not trying to tell the researcher what he or she 'wants to hear', but rather what he or she 'wants to know'. Nevertheless, we cannot say for sure to what extent motivations that may be specific to research participation contributed to cooperative conversational conduct, over and above conversational inferences. Hence, a demand explanation for our findings cannot be ruled out.

Regardless of which particular perspective best accounts for our findings, the 'letterhead effect' observed here bears on a central finding of social psychology, the correspondence bias (Jones, 1979) or the fundamental attribution error (Ross, 1977). This refers to a strong tendency in Western cultures to draw dispositional inferences from behavior rather than situational ones (for a recent review of the correspondence bias, see Gilbert \& Malone, 1995; for a review of cultural differences and similarities, see Norenzayan, Choi, \& Nisbett, in press). Importantly, most research on social inference has been conducted under conditions in which participants are aware that the researcher is a psychologist. Hence, this awareness may have systematically contributed to the dominance of dispositional responses. This awareness, however, is 
unlikely to account for observed cultural differences in attribution, given the likely similarity of participants' assumptions about researchers' interests across cultures.

In conclusion, the present study adds to a growing literature demonstrating that causal attribution, traditionally viewed in social psychology as the outcome of context-free universal principles of reasoning (e.g. Kelley, 1967), is in fact a process which is embedded in the social and communicative context in which it occurs (Hilton, 1990; Hilton \& Slugoski, 1986; Slugoski, et al., 1993). More generally, our findings contribute to the conclusion that the communicative context systematically affects social judgment and reasoning, and that participants bring the tacit assumptions that govern the conduct of conversation in daily life to the research situation (see Hilton, 1995; Kihlstrom, 1995; Schwarz, 1994, 1996). From this perspective, the psychological experiment may not be as artificial and detached from everyday life as some critics would like to claim (e.g. Harré, 1979) — instead, what goes on in the psychological laboratory may be remarkably similar to what happens in daily life (Kihlstrom, 1995). Ironically, however, as researchers, we often miss the extent to which formal features of our research instruments shape participants' responses - a prime example of what Gilbert and Jones (1986) describe as lay perceivers' typical failure to take perceiver-induced constraints on actors' behavior into account.

\section{ACKNOWLEDGEMENTS}

This research was supported by a US National Science Foundation doctoral fellowship to the first author. Thanks to Claire Mercier, Rob Park, Alex Rothman, Miguel Wong, and Nellie Yeretsian for their input and assistance in conducting this research. Bärbel Knäuper, Richard Nisbett, Jeffrey Sanchez-Burks, and three anonymous reviewers provided valuable comments for an earlier version of this manuscript.

\section{REFERENCES}

Bower, G. H., Gilligan, S. G. \& Monteiro, K. P. (1981). Selectivity of learning caused by affective states. Journal of Experimental Psychology: General, 110, 451-483.

Cousins, S. D. (1989). Culture and self-perception in Japan and the U.S. Journal of Personality and Social Psychology, 56, 124-131.

Gilbert, D. T. \& Jones, E. E. (1986). Perceiver-induced constraint: Interpretations of selfgenerated reality. Journal of Personality and Social Psychology, 50, 269-280.

Gilbert, D. T. \& Malone, P. S. (1995). The correspondence bias. Psychological Bulletin, 117, 21-38.

Grice, H. P. (1975). Logic and conversation. In P. Cole \& J. L. Morgan (Eds), Syntax and semantics: Vol 3: Speech acts (pp. 41-58). New York: Academic Press.

Harré, R. (1979). Social being. Oxford: Basil Blackwell.

Higgins, E. T. (1996). Knowledge: Accessibility, applicability, and salience. In E. T. Higgins \& A. Kruglanski (Eds), Social psychology: Handbook of basic principles (2nd edn, pp. 133-168). New York: Guilford Press.

Hilton, D. J. (1990). Conversational processes and causal explanation. Psychological Bulletin, 107, 65-81. 
Hilton, D. J. (1995). The social context of reasoning: Conversational inference and rational judgment. Psychological Bulletin, 118, 248-271.

Hilton, D. J. \& Slugoski, B. R. (1986). Knowledge-based causal attribution: The abnormal conditions focus model. Psychological Review, 93, 75-88.

Jones, E. E. (1979). The rocky road from acts to dispositions. American Psychologist, 34, 107-117.

Kelley, H. H. (1967). Attribution theory in social psychology. In D. Levine (Ed.), Nebraska Symposium on Motivation, 15, pp. 192-230.

Kihlstrom, J. F. (1995). On the validity of psychology experiments. APS Observer, September, pp. $10-11$

Massad, C. M., Hubbard, M. \& Newston, D. (1979). Perceptual selectivity: Contributing process and possible cure for impression perseverance. Journal of Experimental Social Psychology, 15, 513-532.

McCann, C. D. \& Higgins, E. T. (1992). Personal and contextual factors in communication: A review of the 'communication game'. In G. R. Semin \& K. Fiedler (Eds), Language, interaction, and social cognition (pp. 144-172). Newbury Park, CA: Sage.

McGuire, W. J. \& McGuire, C. V. (1988). Content and process in the experience of the self. Advances in Experimental Social Psychology, 21, 97-144.

Miller, J. G. (1984). Culture and the development of everyday social explanation. Journal of Personality and Social Psychology, 46, 961-978.

Morris, M. W. \& Peng, K. (1994). Culture and cause: American and Chinese attributions for social and physical events. Journal of Personality and Social Psychology, 67, 949-971.

Norenzayan, A., Choi, I. \& Nisbett, R. E. Eastern and Western perceptions of causality for social behavior: Lay theories about personalities and situations. In D. A. Prentice \& D. T. Miller (Eds), Cultural divides: The social psychology of intergroup contact. (in press).

Orne, M. (1962). On the social psychology of the psychology experiment. American Psychologist, 17, 776-783.

Ross, L. (1977). The intuitive psychologist and his shortcomings: Distortion in the attribution process. In L. Berkowitz (Ed.), Advances in experimental social psychology (Vol. 10, pp. 174221). New York: Academic Press.

Schwarz, N. (1994). Judgment in social context: Biases, shortcomings, and the logic of conversation. Advances in Experimental Social Psychology, 26, 123-162.

Schwarz, N. (1996). Cognition and communication: Judgmental bias, research methods, and the logic of conversation. Mahwah, NJ: Erlbaum.

Slugoski, B. R., Lalljee, M., Lamb, R. \& Ginsburg, G. P. (1993). Attribution in conversational context: Effect of mutual knowledge on explanation-giving. European Journal of Social Psychology, 23, 219-238.

Sperber, D. \& Wilson, D. (1981). Pragmatics. Cognition, 10, 281-286.

Sperber, D. \& Wilson, D. (1995). Relevance: Communication and cognition (2nd edn). Cambridge, MA: Blackwell.

Srull, T. K. \& Wyer, R. S. (1980). Category accessibility and social perception: Some implications for the study of person memory and interpersonal judgment. Journal of Personality and Social Psychology, 38, 841-856.

Wyer, R. S. \& Srull, T. K. (1989). Memory and cognition in its social context. Hillsdale, NJ: Erlbaum.

Wyer, R. S., Srull, T. K., Gordon, S. E. \& Hartwick, J. (1982). The effects of taking a perspective on recall of prose material. Journal of Personality and Social Psychology, 43, 674-688. 